\title{
Sistema de Classificação de Pacientes na Enfermagem Psiquiátrica: validação clínica*
}

\author{
PATIENT CLASSIFICATION SYSTEM IN PSYCHIATRIC NURSING: CLINICAL VALIDATION \\ SISTEMA DE CLASIFICACIÓN DE PACIENTES EN LA ENFERMERÍA PSIQUIÁTRICA: \\ VALIDACIÓN CLIINICA
}

\author{
Paula Andréa Shinzato Ferreira Martins ${ }^{1}$, Evalda Cançado Arantes ${ }^{2}$, Hideko Takeuchi Forcella ${ }^{3}$
}

\begin{abstract}
RESUMO
A escassez de publicações a respeito de dimensionamento de pessoal na enfermagem psiquiátrica motivou o desenvolvimento de um Instrumento para Classificar o Nível de Dependência na Enfermagem Psiquiátrica, etapa inicial para o estabelecimento da quantidade necessária de profissionais na Equipe de Enfermagem da especialidade. Os objetivos deste estudo buscaram a validação clínica, por meio de testes de confiabilidade e validade do constructo, além da verificação de sua aplicabilidade na prática gerencial do enfermeiro. Foram utilizadas duas amostras, sendo $n=40$ pares de instrumentos preenchidos na Fase 1 da coleta de dados e $n=100$ instrumentos preenchidos na Fase 3 do estudo, tendo sido aplicados diferentes critérios estatísticos, entre eles, o coeficiente Kappa e a correlação de Spearman. O Instrumento para Classificar o Nível de Dependência na Enfermagem Psiquiátrica foi considerado confiável com índices satisfatórios de concordância e o constructo foi validado, determinando o grau de dependência do paciente portador de transtornos mentais.
\end{abstract}

\section{DESCRITORES}

Pessoas mentalmente doentes/ classificação.

Enfermagem psiquiátrica.

Recursos humanos de enfermagem. Reprodutibilidade dos testes. Determinação de necessidades de cuidados de saúde.

\section{ABSTRACT}

The scarcity of publications about personnel dimensioning in psychiatric nursing motivated the development of an Instrument to Classify the Level of Dependence in Psychiatric Nursing, the initial stage for the establishment of the ideal number of professionals in the nursing crew of the specialty. The purpose of this study was to look for the clinical validation through reliability and construct validity tests, as well as to verify its applicability in the management practice of nurses. Two samples were used, of which $n=40$ pairs of instruments filled in Stage 1 of data collection and $n=100$ instruments filled in Stage 3 of the study, with different statistical criteria being applied, among them the Kappa coefficient and the Spearman correlation. The Instrument to Classify the Level of Dependence in Psychiatric Nursing was considered reliable, with good indicators of agreement, and the construct was validated, determining the degree of dependence of mental disorder patients.

\section{KEY WORDS}

Mentally ill persons/classification.

Psychiatric nursing.

Nursing staff.

Reproducibility of results.

Needs assessment.

\section{RESUMEN}

La escasez de publicaciones respecto a la planificación del personal en la enfermería psiquiátrica motivó el desarrollo de un Instrumento para Clasificar el Nivel de Dependencia en la Enfermería Psiquiátrica, etapa inicial para el establecimiento de la cantidad necesaria de profesionales en el Equipo de Enfermería de la especialidad. Los objetivos de este estudio buscaron la validación clínica, por medio de tests de confiabilidad y validez del constructo, además de la verificación de su aplicabilidad en la práctica gerencial del enfermero. Fueron utilizadas dos muestras, siendo $n=40$ pares de instrumentos llenados en la Fase 1 de la recolección de datos y $n=100$ instrumentos llenados en la Fase 3 del estudio, habiendo sido aplicados diferentes criterios estadísticos, entre ellos, el coeficiente Kappa y la correlación de Spearman. El Instrumento para Clasificar el Nivel de Dependencia en la Enfermería Psiquiátrica fue considerado confiable con índices satisfactorios de concordancia y el constructo fue validado, determinando el grado de dependencia del paciente portador de trastornos mentales.

\section{DESCRIPTORES}

Enfermos mentales/classificación.

Enfermería psiquiátrica.

Personal de enfermería.

Reproducibilidad de resultados.

Evaluación de necesidades.

*Extraído da tese "Sistema de classificação de pacientes na especialidade enfermagem psiquiátrica: validação clínica", Escola de Enfermagem, Universidade de São Paulo (EEUSP), 2007. ${ }^{1}$ Enfermeira, especialista, mestre e doutoranda do Programa de Pós-Graduação da Escola de Enfermagem da Universidade de São Paulo, docente de Enfermagem em Saúde Mental e Psiquiátrica da Uni Sant'Anna e da Uni Paulistana. São Paulo, SP, Brasil. paulasfm@ hotmail.com ${ }^{2}$ Enfermeira, mestre e doutora em Enfermagem Psiquiátrica, Docente da Escola de Enfermagem da Universidade de São Paulo, orientadora do Programa de Pós-Graduação em Enfermagem da EEUSP. São Paulo, SP, Brasil. vanibar@usp.br ${ }^{3}$ Enfermeira, mestre e doutora em Enfermagem Psiquiátrica, Docente da Escola de Enfermagem da Universidade de São Paulo, orientadora do Programa de Pós-Graduação em Enfermagem da EEUSP, docente de Enfermagem Psiquiátrica da Faculdade de Ciências Médicas da Santa Casa de São Paulo. São Paulo, SP, Brasil. forcella@usp.br 


\section{INTRODUÇÃO}

Desde o início da década de 90 do século XX, a área de Psiquiatria e Saúde Mental vem evoluindo no Brasil, impulsionada por políticas específicas, aprovações de leis relacionadas ao funcionamento da rede de atendimento, bem como pelos movimentos sociais que buscam a humanização do atendimento recebido pelos indivíduos acometidos por transtornos mentais.

Ao realizar-se a investigação bibliográfica sobre a quantidade necessária de profissionais de enfermagem para atender a demanda de pacientes psiquiátricos internados, depara-se com a escassez de estudos realizados no Brasil sobre o tema dimensionamento de pessoal e na especialidade não foi localizada nenhuma publicação científica a esse respeito.

Pensar na determinação do número ideal de profissionais de enfermagem por pacientes psiquiátricos significa continuar o trajeto de pesquisas na busca de dados que permitirão a composição dos elementos nas fórmulas e equações propostas. Assim, os temas que ainda devem ser pesquisados na especialidade são: os indicadores da qualidade da assistência nas diversas modalidades de atendimento, a quantidade de horas de assistência de enfermagem psiquiátrica, 0 índice de produtividade e 0 tempo efetivo de trabalho da equipe de enfermagem psiquiátrica e 0 absenteísmo e suas causas gerenciais ${ }^{(1)}$.

Em busca de respostas e propostas para solucionar tais inquietações, foi desenvolvido um Sistema de Classificação de Pacientes na especialidade Enfermagem Psiquiátri$\mathrm{ca}^{(2)}$, construindo-se e validando-se um instrumento que determina o nível de dependência dos pacientes acometidos por episódios agudos de transtornos mentais, com base em suas necessidades individualizadas aos cuidados de enfermagem.

O conteúdo do instrumento denominado Instrumento para Classificação do Nível de Dependência em Enfermagem Psiquiátrica (Anexo 1) teve sua validade obtida por intermédio de consenso das opiniões de sete enfermeiros denominados juízes especialistas, que concordaram com a manutenção de 11 indicadores críticos do cuidado.

Como metodologia para validação do conteúdo do instrumento, utilizou-se a técnica Delphi e nessa etapa da investigação, o instrumento foi submetido à validação clínica, ou seja, verificar sua aplicabilidade prática na enfermagem e sua confiabilidade passível de implementação, como parâmetro para melhorar a qualidade da assistência de enfermagem.

Os Sistemas de Classificação de Pacientes foram desenvolvidos no decorrer do século XX. Em 1930, um estudo foi realizado nos Estados Unidos da América (EUA) e identificou as necessidades dos pacientes, encontrando um indicador de horas de enfermagem que, posteriormente, serviria de subsídio a outros estudos ${ }^{(3)}$.

As versões de Sistema de Classificação de Pacientes desenvolvidas na década de 1970 são consideradas simples e manuais, cujos objetivos eram os de atender as necessidades dos pacientes. A partir dos anos de 1980, houve 0 grande desenvolvimento dos modelos, sobretudo, nos EUA onde se buscava o dimensionamento das equipes, chegando, assim, à década de 1990 com instrumentos mais sofisticados e com novos enfoques, entre eles, 0 de custo da assistência ${ }^{(4)}$.

A necessidade de desenvolver métodos para quan-tificar recursos humanos envolve preocupações não só com a qualidade da assistência a ser prestada, bem como a necessidade de adequar os recursos financeiros. Este raciocínio provocou o desenvolvimento de estudos em diversos países como EUA, Canadá, Espanha, entre outros, desde meados da década de 80 do século $X X^{(5-7)}$.

A busca pela melhora da qualidade da assistência investe na crença de que o Sistema de Classificação de Pacientes permitirá o cálculo adequado do dimensionamento da equipe de enfermagem psiquiátrica, cuja finalidade principal será diminuir as vivências manicomiais da internação psiquiátrica, secundárias a serialização e massificação do cuidado que se impõe em muitos locais de tratamento até os dias atuais.

Com base em estudos nacionais e internacionais, já não é possível negar a importância do uso de Sistemas de Classificação de Pacientes na enfermagem, tanto na esfera assistencial como na gerencial, pois os sistemas servem para identificar e dar suporte às demandas do paciente, como ferramenta no processo de tomada de decisão do enfermeiro; identificar e validar os resultados das intervenções de enfermagem, entre outras funções e ainda assim são escassos os estudos publicados para esta população específica(5).

Na Enfermagem Psiquiátrica, os achados científicos sobre Sistemas de Classificação de Pacientes são discretos e se encontrados nota-se que os mesmos discutem apenas a necessidade de desenvolvimento do sistema sem apresentar um modelo real.

Deste modo, não foi localizado outro modelo de Sistemas de Classificação de Pacientes, além do desenvolvido em estudo nacional ${ }^{(2)}$ em 2001, para pacientes portadores de transtornos mentais agudos, decorrentes do nível de dependência da Enfermagem Psiquiátrica.

Em recente publicação, o Resource Utilization Groups - RUG T18 teve sua capacidade preditiva descrita e analisada, após implementação em unidades de internação psiquiátrica, na comunidade de Astúrias, na Espanha(7). 
Conforme os autores citados, em suas considerações finais, a limitação do instrumento que revelou baixa sensibilidade para identificação das necessidades dos pacientes psiquiátricos, foi discutida, e o fator limitante desse fato foi a ausência de um modelo similar e específico para classificar os portadores de transtornos mentais ${ }^{(7)}$.

Os aspectos psíquicos, bem como as necessidades do paciente com transtornos mentais, não haviam sido abordados exclusivamente por nenhum estudo anterior no Brasil. Algumas investigações desenvolvidas incluíram nas categorias e indicadores das necessidades dos pacientes aspectos emocionais, iminência de risco de vida, impossibilidade de comunicar a real necessidade, entre outros termos que, por não serem específicos, não fornecem entendimento preciso à avaliação do paciente.

O Sistema de Classificação de Pacientes na especialidade Enfermagem Psiquiátrica proposto no estudo realizado em 2001, apresenta três categorias dos níveis de dependência de pacientes, assim denominadas: nível de dependência discreta, intermediária e plena aos cuidados de Enfermagem Psiquiátrica(2).

No referido sistema, a classificação do paciente é obtida por intermédio do uso do instrumento desenvolvido que contém 11 indicadores críticos do cuidado com três níveis de gradação cada um. 0 valor da soma dos pontos obtidos assinalados pelo enfermeiro responsável pela avaliação do paciente evidencia seu nível de dependência aos cuidados da enfermagem psiquiátrica.

Assim, os indicadores que compõem o instrumento para Classificação do Nível de Dependência para Enfermagem Psiquiátrica, são: 1)cuidados com a aparência e higiene; 2) expressão do pensamento; 3) humor; interação social; 4) atividades; 5) alimentação e hidratação; 6) sono; 7) medicação; 8) eliminações; 9) sinais vitais e outros controles; 10) problemas e 11) queixas somáticas.

A estrutura gráfica do instrumento elaborado no estu$\mathrm{do}^{(8)}$ foi usada, como referencial no desenvolvimento do instrumento que determina o grau de dependência da Enfermagem Psiquiátrica e todo o processo para elaboração de um Sistema de Classificação de Pacientes foi seguido, conforme o modelo proposto por outro autor ${ }^{(9)}$.

\section{OBJETIVO}

O objetivo deste estudo foi validar o Instrumento para classificação do nível de dependência de Enfermagem Psiquiátrica, proposto em estudo nacional anterior ${ }^{(2)}$, na prática clínica.

\section{MÉTODO}

Estudo quantitativo, do tipo descritivo, exploratório.
A pesquisa foi aprovado pelo Comitê de Ética em Pesquisa da EEUSP sob o parecer do processo no. 482/2005 (CEP-EEUSP), atendendo às exigências da Resolução no . 196/96 do Conselho Nacional de Saúde.

Em seqüência, de posse do parecer favorável do CEP, buscou-se a obtenção da aprovação da Gerência de Enfermagem do local da pesquisa e o consentimento livre e esclarecido dos enfermeiros participantes.

Com a obtenção dos documentos supracitados, testou-se a confiabilidade do instrumento por meio do grau de concordância e validade do constructo, utilizando métodos determinados e aplicando-os em unidades de internação de pacientes psiquiátricos adultos na fase aguda de episódios de transtornos mentais.

\section{Local do estudo e coleta de dados}

Depois de cumpridas as fases iniciais do trajeto metodológico, a validação do instrumento de classificação de pacientes foi realizada nas unidades de internação de um hospital psiquiátrico especializado, localizado na cidade de São Paulo.

Esta instituição atende adultos, portadores de transtornos mentais na fase aguda da doença, disponibilizando 329 leitos psiquiátricos, 269 masculinos e 80 femininos. $\mathrm{Na}$ equipe de Enfermagem, atuam sete enfermeiros e há 100 vagas para Auxiliares de Enfermagem.

Na primeira fase da coleta de dados, cuja finalidade foi a validação de confiabilidade entre avaliadores, 0 instrumento (Anexo 1), foi aplicado em um mesmo paciente por dois enfermeiros simultaneamente. Para tal, antes da aplicação do instrumento, foi explicitado aos enfermeiros que não poderia haver troca de informações entre eles, e o uso do instrumento para classificar os pacientes não significava nenhuma interferência administrativa nas rotinas do serviço dos enfermeiros. Estes aproveitavam 0 momento de transferência de pacientes para outras alas para aplicação do instrumento a ser avaliado. Assim sendo independente do resultado do preenchimento do instrumento, prevaleceria a avaliação do enfermeiro na decisão assistencial do paciente, permitindo que 0 sujeito da pesquisa coletasse os dados classificando os pacientes quanto ao nível de dependência, sem necessariamente, haver interferência em seu processo de tomada de decisão quanto à transferência do paciente.

Por meio da técnica de amostragem não probabilística denominada por conveniência ou tendenciosa, pautada nas possibilidades e condições dos pares de enfermeiros, 80 instrumentos foram preenchidos, tendo sido avaliados 40 pacientes por dois enfermeiros cada.

É importante ressaltar que os pares de enfermeiros que preencheram os instrumentos simultaneamente, tinham conhecimentos semelhantes sobre os pacientes avaliados em razão da condição de atuação desses profis- 
sionais, pois ao enfermeiro compete decidir sobre a possibilidade de transferência do paciente às demais unidades de internação hospitalar.

As informações obtidas na Fase 2 da coleta de dados permitiram descrever o perfil dos sujeitos da pesquisa, bem como obter a opinião dos mesmos a respeito da aplicação do instrumento.

Assim, dos sete enfermeiros que anuíram participar do estudo, três tiveram oportunidade de atender as solicitações da pesquisadora, em relação à aplicação do Instrumento para Classificar o Nível de Dependência de Pacientes Psiquiátricos, destes dois eram do sexo feminino e um do sexo masculino, a média de idade foi de 38 anos e o tempo de formado variou entre dois e 16 anos.

Quanto à formação na área de enfermagem, dois possuíam pós-graduação latu-senso e um stricto-senso na Enfermagem Psiquiátrica.

Dentre os sujeitos, todos atuavam na Enfermagem Psiquiátrica com variação de tempo entre dez meses e 16 anos, e dois desenvolvem atividades no ensino e na pesquisa na própria especialidade.

\section{Tratamento dos dados}

Nesta etapa do estudo as seguintes definições foram consideradas:

Confiabilictade é o grau de coerência com o qual um instrumento mede 0 atributo, sendo possível afirmar quanto menor a variação produzida pelo instrumento, em repetidas mensurações de um atributo, maior será sua confiabi-lidade, expressando se este poderá ser replicado com garantia de resultados semelhantes e precisos ${ }^{(9-10)}$.

Concordânciaé o grau habitualmente expresso em porcentagem, com que diferentes avaliadores tendem a fazer os mesmos julgamentos sobre o que está sendo avaliado ${ }^{(10)}$.

Neste estudo, o teste de confiabilidade entre-avaliadores ou objetiva foi selecionado e adotou-se o teste estatístico do Coeficiente de Kappa para medir a concordância entre eles.

A concordância total entre as respostas dos enfermeiros resulta em um valor de Kappa $=1$, enquanto a total discordância entre as respostas, em um valor de Kappa $=0$, lembrando que 0 valor $=-1$ representaria a inversão das concordâncias.

Procedeu-se, então, a aplicação do coeficiente de Kappa nos 11 indicadores críticos listados no Instrumento para Classificação do Nível de Dependência de Enfermagem Psiquiátrica.

Todos os cruzamentos foram apresentados dois a dois com os respectivos coeficientes Kappa, objetivando determinar a intensidade da concordância nas classificações e no escore geral.
A terceira e última fase da coleta de dados buscou a validação de constructo do instrumento, que se realizou com sua aplicação de forma numericamente expressiva, no mesmo hospital, campo de pesquisa.

Empregou-se a técnica amostral aleatória ou por conveniência, com a escolha de $30 \%$ da população, totalizando 98,7 pacientes, aqui arredondados para 100 indivíduos, que foram avaliados para posterior aplicação do instrumento.

Nesta fase, duas enfermeiras foram sujeitos da pesquisa, sendo uma delas a pesquisadora.

Validade pode ser definida, como o grau no qual um instrumento mede aquilo que se propõe medir ${ }^{(10)}$, sendo ainda mais desafiador estabelecer a validade do que a confiabilidade de um instrumento que pode ser considerado confiável, mas, não válido ou válido e não confiável, acreditando que o ideal seja um instrumento válido e confiável ${ }^{(9-10)}$.

Constructos são valores considerados para análise de um conceito ou idéia e costumam ser explicados em termos de outros conceitos. Conseqüentemente, o pesquisador precisa fazer previsões a respeito de como 0 constructo funcionará. Logo, sua validade não é provada ou estabelecida e, sim, apoiada em evidências ${ }^{(11)}$.

No presente estudo, o constructo validado referiu-se ao nível de dependência dos pacientes portadores de transtornos mentais agudos, ao cuidado de Enfermagem Psiquiátrica, medido com base no Instrumento para Classificar o Nível de Dependência em Enfermagem Psiquiátri$\mathrm{ca}^{(2)}$, buscando identificar se 0 instrumento que se propõe a medir o grau de dependência do paciente aos cuidados de enfermagem alcança seu objetivo, determinando a complexidade assistencial do paciente avaliado.

Instrumentos de classificação de pacientes constituem-se em escalas nominais e, assim, neste estudo, 0 Coeficiente de Spearman foi adotado para validar seu constructo.

\section{RESULTADOS}

Os dados deste estudo foram coletados em três fases distintas, sendo a primeira amostra de $n=40$ pares de instrumentos buscavam a confiabilidade e o grau de concordância destes para classificar o nível de dependência de Enfermagem Psiquiátrica.

A segunda fase da coleta de dados consistiu na apresentação e discussão das opiniões emitidas pelos enfermeiros, sujeitos da pesquisa no primeiro momento sobre a utilização do instrumento.

Já os dados do terceiro momento da coleta de dados compreenderam outros 100 instrumentos preenchidos, buscando a validação do constructo proposto. 
Desta forma, os dados coletados para as Fases 1 e 3, inicialmente, foram transcritos e tabulados em planilhas do aplicativo "Excel" na versão Office 2003, procedendose ao registro das informações apontadas pelos enfermeiros, sujeitos da pesquisa.
0 critério adotado de análise foi o teste estatístico para aplicação do Coeficiente de Kappa, utilizado para avaliar o grau de concordância dos escores totais obtidos pelos enfermeiros, buscando a confiabilidade do instrumento.

Tabela 1- Kappa Geral - Geral das comparações entre as respostas dos enfermeiros - São Paulo - 2006

\begin{tabular}{l|l|l|l|l|l}
\hline \multicolumn{1}{c}{ Indicadores } & \multicolumn{3}{c}{ Discordância } & \multicolumn{3}{c}{ Medidas de Comparabilidade } \\
Variável Comparada & 4 & 10 & 0,765 & $<0,0001$ & $\begin{array}{l}\text { Sup. } 1,0 \\
\text { Inf. } 0,464\end{array}$ \\
\hline 1) Aparência e Higiene & 13 & 32,5 & 0,517 & $<0,0001$ & $\begin{array}{l}\text { Sup. } 0,748 \\
\text { Inf. } 0,286\end{array}$ \\
\hline 2) Expressão de Pensamento & 13 & 32,5 & 0,47 & $<0,01$ & $\begin{array}{l}\text { Sup. } 0,704 \\
\text { Inf. } 0,236\end{array}$ \\
\hline 3) Humor e Afeto & 21 & 52,5 & 0,171 & 0,128 & $\begin{array}{l}\text { Sup. } 0,391 \\
\text { Inf. } 0,0049-\end{array}$ \\
\hline 4) Atividades & 8 & 20 & 0,659 & $<0,001$ & $\begin{array}{l}\text { Sup. } 0,899 \\
\text { Inf. } 0,419\end{array}$ \\
\hline 5) Interação Social & & & & & \\
\hline 6) Alimentação e Hidratação & 3 & 7,5 & 0,725 & $<0,001$ & $\begin{array}{l}\text { Sup. } 1,0 \\
\text { Inf. } 0,416\end{array}$ \\
\hline 7) Sono & 9 & 22,5 & 0,397 & 0,006 & $\begin{array}{l}\text { Sup. } 0,678 \\
\text { Inf. } 0,116\end{array}$ \\
\hline 8) Medicamento & 4 & 10 & 0,736 & $<0,001$ & $\begin{array}{l}\text { Sup. } 1,0 \\
\text { Inf. } 0,468\end{array}$ \\
\hline 9) Eliminações & 6 & 15 & 0,624 & $<0,001$ & $\begin{array}{l}\text { Sup. } 0,934 \\
\text { Inf. } 0,314\end{array}$ \\
\hline 10)Sinais Vitais e Outros Controles & 8 & 20 & 0,31 & 0,046 & $\begin{array}{l}\text { Sup. } 0,616 \\
\text { Inf. } 0,005\end{array}$ \\
\hline 11)Queixas e Problemas Somáticos & 13 & 32,5 & 0,312 & 0,018 & $\begin{array}{l}\text { Sup. } 0,57 \\
\text { Inf. } 0,054\end{array}$ \\
\hline
\end{tabular}

Fonte: Martins ${ }^{(12)}$

${ }^{1}$ Indica o valor máximo de estatística p em relação ao nível de significância estabelecido (quanto menor o valor de Kappa, maior o comprometimento desta estatística, pois ela se aproxima ou até ultrapassa o nível de $5 \%=0,005$ );

${ }^{2}$ Indica os valores máximos (superior) e mínimos (inferior) do valor de Kappa na questão estudada. Mede a dispersão dos valores de Kappa.

Ao se analisar os dados apresentados na Tabela 1, 0 nível de concordância Kappa, entre os enfermeiros variou de 0,171 a 0,765, no geral, identifica-se que houve concordância entre as respostas de todos os indicadores, tomando-se como base a escala de dos autores ${ }^{(13)}$ que apresenta os diferentes níveis de concordância.

0 indicador 1. Cuidados com Aparência e Higiene apresentou Kappa $=0,765$, com excelente concordância entre as respostas e $5 \%$ de significância.

Ao analisar-se o Intervalo de Confiança das respostas foi possível perceber que este variou de 0,464 a 1,0, o que é a concordância plena.

Ao analisar os níveis de concordância, a dependência discreta (1) apareceu com destaque, enquanto a dependência plena (3) não apresentou nenhuma incidência.

Aqui, a concordância alcançou o índice máximo pelo fato do indicador 1. - Cuidados com a Aparência e Higiene, avaliar a necessidade de orientação, estímulos e supervisão da equipe de enfermagem quanto à possibilidade, capacidade e adequação do paciente realizar cuidados pessoais como, por exemplo, a escolha de roupas, ornamentos e uso do chuveiro.

Assim, no momento da avaliação, existiu a possibilidade real do enfermeiro ao observar o paciente, decidir sobre seu nível de dependência, pois as evidências das necessidades dele tornam-se objetivas, claras e até ululantes.

Já o indicador 4. Atividades apresentou o menor grau de concordância entre as respostas por meio do teste de Kappa $=0,171$.

O Intervalo de Confiança, de $95 \%$, representa que os valores variaram de $-0,049$ a 0,391. Para o nível 2 (dependência intermediária) deste indicador, o valor $(-0,049(0))$ mostra que não houve concordância entre os enfermeiros, ao passo que no nível 3 (dependência plena) 0 valor de Kappa $=0,435$ resulta na concordância que é mediana.

A diferença de porcentual entre as respostas obtidas neste indicador, já tinha sido observada e apresentada anteriormente, quando foram constatadas 21 respostas divergentes das 40 obtidas.

Assim, sugere-se uma análise mais apropriada em relação ao que significam os níveis 1, 2 e 3 e 0 entendimento dos enfermeiros ao classificar pacientes em função desse indicador no instrumento analisado, pois observa-se que embora não haja nenhuma divergência grave, o teste Kappa sensibilizou-se com o fato de haver ou não divergência e não com o tipo desta. 
Este indicador aborda em seus três níveis de dependência a participação do paciente nas atividades propostas, avaliando sua aceitação ou recusa sua permanência e, até mesmo, a adequação de seu comportamento no evento.

Destaca-se a influência da subjetividade de cada enfermeiro, pois a classificação desse indicador requer a avaliação da qualidade e do desempenho do paciente; dentre as causas das variações, habita a possibilidade pela qual cada um dos observadores adota um critério para sua decisão e há diferença quanto às formas de interpretar as informações obtidas ${ }^{(14)}$.

A subjetividade pode ser definida, como: caráter de todos os fenômenos psíquicos, como fenômenos da consciência que o sujeito relaciona consigo mesmo e chama de meus ${ }^{(15)}$. Assim, pode-se afirmar que o enfermeiro ao avaliar o paciente neste 40 indicador - Atividades conceitua 0 paciente naquilo que ele, enfermeiro, pode ver e, conseqüentemente, torna-se necessário evidenciar que esta avaliação seja a mais objetiva possível, questionando o paciente e conhecendo-o para melhor avaliá-lo.

Outro aspecto deve-se à necessidade de conhecimento prévio sobre do paciente pelo enfermeiro, pois este indicador refere-se a um comportamento do cotidiano do paciente, não sendo possível classificá-lo apenas levando em consideração 0 exato momento da avaliação do enfermeiro.

Como o instrumento proposto busca avaliar e determinar o grau de dependência aos cuidados de enfermagem psiquiátrica, a maioria dos indicadores críticos para o cuidado aborda os aspectos comportamentais e as inadequações do paciente provocadas por esses comportamentos.

A discussão sobre as discordâncias entre as respostas dos enfermeiros será sempre justificada pela possibilidade da interpretação subjetiva das necessidades do paciente frente à avaliação do sujeito avaliador do processo. Assim, deve-se buscar apoio nas concordâncias, evidenciando a possível confiabilidade do instrumento psiquiátrico.
Os resultados de Kappa obtidos para os indicadores 2, 3, 5, 6, 7, 8, 9, 10 e 11 apontaram índice de concordância mediano, o que é aceitável para adotar um instrumento, como ferramenta para classificar pacientes.

O outro critério estatístico consistiu na aplicação do teste de correlação de Spearman.

Esta etapa da análise teve por objetivo verificar a validação do constructo (ou da construção) do instrumento que permite concluir se as informações fornecidas ou observadas podem ser utilizadas com confiança pelos enfermeiros psiquiátricos com base na correlação entre os indicadores listados.

Assim sendo, em que pese a característica subjetiva do enfermeiro observador ao responder 0 instrumento à medida que visita cada um dos pacientes e a possível diferença dentre cada grau discreto, intermediário ou pleno de dependência, optou-se por um teste que praticamente ignorasse essas fundamentais diferenças e observou-se se existe diferença significativa dentre os blocos de respostas em cada um dos 11 indicadores.

Como onze são os indicadores, ao compará-los dois a dois, ter-se-iam 55 combinações diferentes de opção.

Mediante a aplicação do software, foi calculado se havia associação ou correlação entre as respostas e ao compará-las, dentre as 55 comparações possíveis, optouse por apresentar algumas delas, incluindo-se aquelas das extremidades máxima e mínima.

Considerando-se cinqüenta e cinco possibilidades diferentes de respostas, tomou-se como parâmetro as comparações, cujos resultados e respostas foram próximos, por exemplo, os indicadores $9 \times 10$ e as comparações entre resultados com maior quantidade de divergências como, por exemplo, indicadores $2 \times 10$ apresentadas na Tabela 2 .

Se houver significância estatística dentre estas situações extremas, todas as demais, nesse intervalo, também, terão associação entre as respostas e correlação positiva (forte ou fraca), entre elas.

Tabela 2 - Analítico (resumido) dos valores do coeficiente de Spearman obtidos na comparação entre os resultados dos 100 pacientes em relação aos graus de dependência apresentados na coleta de dados - São Paulo - 2006

\begin{tabular}{|c|c|c|c|c|c|c|c|}
\hline Indicador $\mathrm{x}$ indicador & $9 \times 10$ & $\ldots$ & $2 \times 3$ & $\ldots$ & $5 \times 11$ & $\ldots$ & $2 \times 10$ \\
\hline Coeficiente Spearman & 0,998 & & 0,992 & & 0,9107 & & 0,8632 \\
\hline (rs) em $\%$ & $99,81 \%$ & & $99,18 \%$ & & $91,07 \%$ & & $86,32 \%$ \\
\hline
\end{tabular}

Fonte: Martins ${ }^{(12)}$

Dentre as cinqüenta e cinco combinações de comparação, tomando-se as duas maiores divergências quanto aos níveis $(1,2$ e 3$)$ foram obtidos, como coeficiente de Spearman (rs) e cálculo da estatística t de Student, valores que não apresentam diferença significativa.
Portanto, o constructo ou construção do instrumento não apresenta divergências importantes do ponto de vista da estatística, sob análise da estatística descritiva, de medidas de dispersão em torno da média aritmética e da estatística Indutiva ou Inferencial pela análise por meio dos testes Qui- Quadrado, estatística t de Student e Coeficiente de Correlação de Spearman, evidenciando sua validade.

$\begin{array}{rrr}\text { Rev Esc Enferm USP } & \text { Sistema de classificação de pacientes na } \\ 2008 ; 42(2): 233-41 . & \text { Enfermagem Psiquiátrica: validação clínica } \\ \text { www.ee.usp.br/reeuspl } & \text { Martins PASF, Arantes EC, Forcella HT }\end{array}$




\section{CONCLUSÕES}

O Instrumento para Classificação do Nível de Dependência em Enfermagem Psiquiátrica obteve sua validação clínica para implementação na prática assistencial da especialidade, denotando a possibilidade de melhoria do atendimento do paciente, tanto na qualidade do serviço como na evidência do dimensionamento de pessoas, sendo ainda necessário estudar outros aspectos relacionados ao tema.

Os primeiros testes estatísticos demonstraram que 0 instrumento é confiável, obtendo grau de concordância moderado.

Dentre os indicadores mais significativos para a especialidade, encontra-se o de Cuidados com a Aparência e Higiene. Desta forma, deve-se concentrar a atenção no desenvolvimento da habilidade de observação dos enfermeiros, adotando critérios para tal.

Para tornar público um Sistema de Classificação de Pacientes, o pesquisador deve garantir a validade do conteúdo e a do constructo, além da confiabilidade do método, permitindo à comunidade científica sua adoção, como método, ou mesmo, como material de referência no desenvolvimento de novos modelos.

É sabido que, para a prática de Enfermagem Psiquiátrica, outros modelos de Sistema de Classificação de

\section{REFERÊNCIAS}

1. Martins PASF, Forcella HT. Dimensionamento da equipe de enfermagem psiquiátrica: o quê ainda precisamos estudar? Rev Acad Enferm. 2004;2(2):34-7.

2. Martins PASF. Sistema de Classificação de Pacientes na especialidade Enfermagem Psiquiátrica [dissertação]. São Paulo: Escola de Enfermagem, Universidade de São Paulo; 2001.

3. Rodrigues Filho J. Sistema de Classificação de Pacientes. Parte I: dimensionamento de pessoal de enfermagem. Rev Esc Enferm USP. 1992;26(3):395-404

4. Malloch K, Neeld AP, McMurry C, Meeks L, Wallach M, Williams $S$, et al. Patient classification systems. Part 2: the third generation. J Nurs Adm. 1999;29(9):33-42.

5. Wrona-Sexton S. Patient Classification Systems: another perspective. Nurs Manage. 1992;23(12):38-9.

6. Perroca MG. Sistema de Classificação de Pacientes: construção e validação de um instrumento [dissertação]. São Paulo: Escola de Enfermagem, Universidade de São Paulo; 1996.

7. Iglesias C, Alonso Villa MJ. A System of Patients Classification inlong-term psychiatric inpatients: Resource Utilizantion Groups T-18 (RUG T-18). J Psychiatr Ment Health Nurs. 2005;12(1):33-7.

8. Romero EA, Araújo ES, Watanabe MY, Lisboa MAPLP, Abud $\mathrm{MH}$, Barroso RV, et al. Proposta de um instrumento para a classificação dos cuidados de enfermagem quanto ao grau de dependência. In: Anais do 50 ENFTEC; 1994; São Paulo. p. $482-5$
Pacientes devem ser desenvolvidos para atender a diversidade de modalidades assistenciais, como os Centros de Atenção Psicossocial, as residências terapêuticas, os hospitais-dia e mesmo as unidades psiquiátricas dentro de hospitais gerais.

Para avaliação de pacientes na busca da classificação do nível de dependência em Enfermagem Psiquiátrica, indica-se, além da adoção de um instrumento validado, 0 treinamento específico, caso o enfermeiro não seja especialista.

Deve-se considerar, ainda, o fato dos indicadores apresentarem certo grau de subjetividade, pois alguns se relacionam ao comportamento do paciente e podem sofrer a interferência do ambiente, das relações enfermeiro-paciente, entre outras possibilidades.

Assim, a tônica da adoção de um Sistema de Classificação de Pacientes na especialidade Enfermagem Psiquiátrica é a capacitação dos enfermeiros em temas, como comunicação terapêutica, exame psíquico de enfermagem, psicopatologia e alterações de comportamento e, em destaque, as questões relacionadas à subjetividade na área, bem como a aproximação dos profissionais dos pacientes atendidos.

Acredita-se que, com a validação clínica deste instrumento, será possível contribuir para o caminho que deve manter seu percurso na busca de soluções aos desafios propostos pelo dimensionamento da Enfermagem Psiquiátrica.

9. Perroca MG. Instrumento de classificação de pacientes de PERROCA: validação clínica [tese]. São Paulo: Escola de Enfermagem, Universidade de São Paulo; 2000.

10. Andrade AG, Scalco MZ. Conceitos de validade e confiabilidade de um instrumento para avaliação de tratamentos de alcoolistas. J Bras Psiq. 1989;38(1):41-5.

11. Cassiani SHB. A coleta de dados nas pesquisas em enfermagem: estratégias, validades e confiabilidade [dissertação]. Ribeirão Preto: Escola de Enfermagem de Ribeirão Preto, Universidade de São Paulo; 1987.

12. Martins PASF. Sistema de Classificação de Pacientes na Especialidade Enfermagem Psiquiátrica: validação clínica [tese]. São Paulo: Escola de Enfermagem, Universidade de São Paulo; 2007.

13. Landis JR, Koch GG. The measurement of observer agrrement for categorical data. Biometrics. 1977;33(1):159-75.

14. Menezes PR. Validade e confiabilidade das escalas de avaliação em psiquiatria. Rev Psiquiatr Clin [periódico na Internet]. 1998 [citado 2006 nov. 13];25(5). Disponível em: http:// www.hcnet.usp.br/ipq/revista/r255/conc 255b .htm

15. Machado AL, Colvero LA. O cuidado de enfermagem: olhando através da subjetividade. Acta Paul Enferm. 1999;12(2):66-72. 
Anexo I

Instrumento para Classificação do Nível de Dependência em Enfermagem Psiquiátrica

\begin{tabular}{|c|c|c|}
\hline \multicolumn{2}{|c|}{ Nome do Paciente: } & Ocupação: \\
\hline \multicolumn{2}{|c|}{ Idade: } & Data de Internação: __/_/_ \\
\hline \multicolumn{3}{|c|}{ 1. Cuidados com a Aparência e Higiene } \\
\hline 1 & Discreta & $\begin{array}{l}\text { Necessita apenas de orientação e supervisão para realizar as } \\
\text { atividades de rotina como: uso do chuveiro, guarda de roupas e } \\
\text { pertences e higiene adequada. Faz uso adequado de vestimentas e } \\
\text { ornamentos. }\end{array}$ \\
\hline 2 & Intermediária & $\begin{array}{l}\text { Necessita de orientação, estímulos verbais e auxílio para higiene } \\
\text { adequada. Demonstra algum desinteresse por sua } \\
\text { aparência.Abusa de ornamentos. }\end{array}$ \\
\hline 3 & Plena & $\begin{array}{l}\text { Negligente quanto à aparência, veste-se de forma inadequada e ou } \\
\text { bizarra, necessita de ajuda para tomar banho, escovar os dentes e } \\
\text { realizar higiene intima. }\end{array}$ \\
\hline \multicolumn{3}{|r|}{ 2. Expressão do Pensamento } \\
\hline 1 & Discreta & $\begin{array}{l}\text { Demonstra crítica e juízo preservados. Responde às solicitações. } \\
\text { Mantém discurso em tom de voz normal e conteúdo adequado. }\end{array}$ \\
\hline 2 & Intermediária & $\begin{array}{l}\text { Mantém tom de voz elevado ou diminuído. Responde } \\
\text { sucintamente às solicitações. Nota -se prejuízo da crítica e } \\
\text { julgamento, mantém discurso acelerado, mudando várias vezes de } \\
\text { assunto sem encerrar o anterior, fala aparentemente sozinho; } \\
\text { mas, quando solicitado, consegue manter um discurso coerente. }\end{array}$ \\
\hline 3 & Plena & $\begin{array}{l}\text { Apresenta idéias delirantes, idéias que expressam alucinações, } \\
\text { denotando com certa freqüência grande incômodo resultante de } \\
\text { tais sintomas, expressa idéias de agitação, fuga ou suicídio, não } \\
\text { responde às solicitações. M antém-se em mutismo ou apesar das } \\
\text { alterações, não é capaz de expressálas. }\end{array}$ \\
\hline \multicolumn{3}{|r|}{ 3. Humor e Afeto } \\
\hline 1 & Discreta & Mantém humor eutímico. \\
\hline 2 & Intermediária & $\begin{array}{l}\text { Demonstra certa indiferença, chora sem motivo aparente e com } \\
\text { facilidade; não expressa seus sentiment os, faz demonstrações } \\
\text { afetivas inadequadas, às vezes alegre, às vezes triste. }\end{array}$ \\
\hline 3 & Plena & $\begin{array}{l}\text { Incapacidade para manejar seus sentimentos excessivamente } \\
\text { alegre ou triste, desinteressado de tudo, irrita } \\
\text { muda bruscamente de estado de humor. Refe } \\
\text { morrer. }\end{array}$ \\
\hline
\end{tabular}

\begin{tabular}{|c|c|c|}
\hline \multicolumn{3}{|r|}{ 4. Atividades } \\
\hline 1 & Discreta & $\begin{array}{l}\text { Aceita participar das atividades individuais e grupais, colaborador } \\
\text { e afetivo; procura ocupações espontaneamente, termina o que } \\
\text { inicia e executa-as adequadamente. }\end{array}$ \\
\hline 2 & Intermediária & $\begin{array}{l}\text { Participa de ati vidades, apenas quando é estimulado, mantendo - } \\
\text { se isolado dos demais; não consegue permanecer integralmente } \\
\text { nas atividades, não termina o que inicia, tem dificuldade de } \\
\text { entrosamento durante as atividades. }\end{array}$ \\
\hline 3 & Plena & $\begin{array}{l}\text { Recusa participar de qualquer atividade, apesar de conhecê -las; } \\
\text { fica parado (completamente inativo), não permanece nas } \\
\text { atividades. }\end{array}$ \\
\hline
\end{tabular}

\begin{tabular}{|l|l|l|}
\hline \multicolumn{2}{|l|}{} \\
\hline 2 & \multirow{2}{*}{} & Discreta \\
& & \\
\hline 3 & & \\
& & \\
\hline
\end{tabular}

\section{Interação Social}

Colaborador, procura interagir espontaneamente.

Mantém-se isolado dos demais; indeciso, tenta seduzir e manipular os demais; anda sozinho de um lado para outro; tem dificuldade no entrosamento e no cotidiano do manejo das relações familiares e social; quando solicitado, interage ou apenas responde às solicitações.

Hostil e ameaçador; não tolera frustrações; muito dependente dos demais; fica parado (completamente inativo); não colabora; fuma em demasia; furta pertences dos demais; aborda familiares de outros pacientes durante a visita; negligencia suas responsabilidades. 
continuação...

\begin{tabular}{|c|c|c|}
\hline \multicolumn{3}{|r|}{ 6. Alimentação / Hidratação } \\
\hline 1 & Discreta & $\begin{array}{l}\text { Aceita adequadamente as refeições e hidratação; mantém hábitos } \\
\text { adequados durante as refeições, considerando -se as diferenças } \\
\text { culturais. }\end{array}$ \\
\hline 2 & Intermediária & $\begin{array}{l}\text { Ingere quantidade insuficiente de alimentos; exige dieta especial } \\
\text { (terapêutica); quando estimulado e orientado alimentase; mantém } \\
\text { alguns hábitos inadequados durante as refeições, considerando-se } \\
\text { as diferenças culturais. }\end{array}$ \\
\hline 3 & Plena & $\begin{array}{l}\text { Não se alimenta sozinho; tem dificuldade para mastigar ou } \\
\text { deglutir; recusa as refeições; ingere quantidade excessiva de } \\
\text { alimentos; mantém -se inadequado durante as refeições; realiza } \\
\text { ações purgativas, após as refeições. }\end{array}$ \\
\hline \multicolumn{3}{|r|}{ 7. Sono } \\
\hline 1 & Discreta & Dorme regularmente à noite. \\
\hline 2 & Intermediária & $\begin{array}{l}\text { Dorme durante o dia; não dorme à noite mas permanece em seu } \\
\text { leito; só dorme após ser medicado }(\mathrm{s} / \mathrm{n}) \text {. }\end{array}$ \\
\hline 3 & Plena & $\begin{array}{l}\text { Dorme e queixa -se de que não dormiu; nã o dorme dia e noite e } \\
\text { torna-se inquieto e agitado; sonâmbulo; não dorme mesmo depois } \\
\text { de medicado uma vez; dorme além do normal tanto de dia como à } \\
\text { noite. }\end{array}$ \\
\hline
\end{tabular}

\begin{tabular}{|c|c|c|}
\hline \multicolumn{3}{|r|}{ 8. Medicação } \\
\hline 1 & Discreta & $\begin{array}{l}\text { Aceita sua medicação; quase sempre conhece os medicamentos } \\
\text { que usa, bem como seus efeitos; é possivel responsabilizá -lo pela } \\
\text { própria medicação. }\end{array}$ \\
\hline 2 & Intermediária & $\begin{array}{l}\text { Aceita sua medicação após orientação e abordagem; apresenta } \\
\text { sintomas de efeitos colaterais e indesejáveis da medicação; } \\
\text { desconhece os medicamentos que usa, bem como seus efeitos; } \\
\text { demonstra certa insatisfação ou medo dos medicamentos; } \\
\text { eventualmente, procura por informações sobre a medicação. }\end{array}$ \\
\hline 3 & Plena & $\begin{array}{l}\text { Faz tentativas de esconder sua medicação; recusa os } \\
\text { medicamentos; necessita de medicações injetáveis; solicita } \\
\text { medicamentos a todo o momento. }\end{array}$ \\
\hline \multicolumn{3}{|r|}{ 9. Eliminações } \\
\hline 1 & Discreta & As eliminações estão presentes; tem controle esfincteriano. \\
\hline 2 & Intermediária & $\begin{array}{l}\text { Suas eliminações não são diárias ou são excessivas; tem controle } \\
\text { esfincteriano; apresenta obstipação ou elimin ações intestinais } \\
\text { líquidas; apresenta incontinência urinária decorrente do uso de } \\
\text { medicações. }\end{array}$ \\
\hline 3 & Plena & Não tem controle esfincteriano; faz uso inadequado do sanitário. \\
\hline
\end{tabular}

\begin{tabular}{|c|c|c|}
\hline \multicolumn{3}{|c|}{ 10. Sinais Vitais e outros Controles } \\
\hline 1 & Discreta & Necessita de verificação sistematizadamente. \\
\hline 2 & Intermediária & $\begin{array}{l}\text { Necessita de verificações de acordo com a evolução clínica, } \\
\text { sintomatológica ou queixas. }\end{array}$ \\
\hline 3 & Plena & $\begin{array}{l}\text { Necessita de controle de sinais vitais, hídrico, de débito urinário, } \\
\text { glicemia, ou outros, várias vezes ao dia; apresenta disfunções } \\
\text { clínicas não psiquiátricas (HAS; Diabetes Mellitus; outras). }\end{array}$ \\
\hline \multicolumn{3}{|c|}{ 11. Queixas e Problemas Somáticos } \\
\hline 1 & Discreta & Nega queixas somáticas. \\
\hline 2 & Intermediária & $\begin{array}{l}\text { Refere queixas relativas ao tratamento medicamentoso, de sinais e } \\
\text { sintomas crônicos ou outras. }\end{array}$ \\
\hline 3 & Plena & $\begin{array}{l}\text { Refere queixas de sintomas agudos de disfunções fisiológicas ou } \\
\text { clínicas; apresenta sinais e sintomas de patologias clínicas. }\end{array}$ \\
\hline \multicolumn{3}{|c|}{ Classificação - Nivel de Dependência } \\
\hline \multicolumn{3}{|c|}{$\begin{array}{l}\text { Diagnóstico de Enfermagem: } \\
\text { Diagnóstico Clínico: }\end{array}$} \\
\hline Data: & L & Enf $^{\circ}$. \\
\hline \multicolumn{3}{|r|}{ Classificação por Nivel de Dependência } \\
\hline & Pontuação & Descrição \\
\hline & 11 à 18 & Grau de Dependência Discreta \\
\hline & 19 à 26 & Grau de Dependência Intermediária \\
\hline & 27 à 33 & Grau de Dependência Plena \\
\hline
\end{tabular}

Fonte: Martins (12) 